\title{
Comparison of Continuous and Intermittent IV Infusion of Vancomycin: Systematic Review
}

\author{
Sally S K Man, Roxane R Carr, and Mary H H Ensom
}

\begin{abstract}
Background: There is some evidence that administration of vancomycin by continuous infusion has pharmacokinetic and pharmacodynamic advantages over traditional intermittent dosing. Whether these advantages translate into clinical efficacy remains controversial.
\end{abstract}

Objective: To review the literature comparing continuous infusion of vancomycin and conventional intermittent IV dosing in terms of efficacy and safety.

Methods: A literature search was conducted in the PubMed/MEDLINE and Embase databases and the Cochrane Central Register of Controlled Trials, and by means of the Google search engine, and the reference lists of pertinent articles were searched manually. All human studies published in English or French that evaluated vancomycin given by continuous and intermittent IV infusion were reviewed. Articles that did not include a comparator arm and those that assessed continuous and intermittent intraperitoneal infusions were excluded. The level of evidence of each study was categorized according to the US Preventive Services Task Force rating scale.

Results: In total, 9 studies were identified: 1 in a pediatric population and 8 in adult populations. Of the 3 studies with the highest quality of evidence (level I), one demonstrated pharmacodynamic advantages with continuous infusion of vancomycin. Of the 6 studies representing a moderate level of evidence (level II), 3 also favoured continuous infusion in terms of pharmacokinetic and pharmacodynamic outcomes, but the findings in terms of clinical outcomes were mixed.

Conclusions: Current evidence evaluating the pharmacokinetic and pharmacodynamic advantages and clinical efficacy of continuous versus intermittent vancomycin infusions is inconsistent and does not support the routine use of continuous infusion for the treatment of multidrugresistant gram-positive infections.

Key words: vancomycin, continuous infusion, intermittent infusion

Can J Hosp Pharm 2010;63(5):373-381

\section{RÉSUMÉ}

Contexte : Selon certaines données, l'administration de vancomycine en perfusion i.v. continue aurait des avantages pharmacocinétiques et pharmacodynamiques par rapport à la perfusion i.v. intermittente traditionnelle. Cependant, il existe toujours une controverse à savoir si ces avantages se traduisent par une efficacité clinique.

Objectif : Passer en revue la littérature sur l'efficacité et l'innocuité de la perfusion continue de vancomycine par rapport à la perfusion intermittente traditionnelle.

Méthodes: Une recherche bibliographique a été effectuée dans les bases de données PubMed/MEDLINE et Embase et dans le Registre central Cochrane des essais randomisés, et également au moyen du moteur de recherche Google et de l'examen manuel des listes de référence des articles pertinents extraits. Toutes les études effectuées sur des humains publiées en anglais ou en français évaluant la vancomycine administrée par perfusion i.v. intermittente et continue ont été analysées. Les articles qui n'avaient pas de groupe de comparaison et ceux qui évaluaient les perfusions intrapéritonéales intermittentes et continues ont été exclus. Le niveau de données probantes pour chaque étude a été classé selon l'échelle de notation du US Preventive Services Task Force.

Résultats : Au total, neuf études ont été recensées : une dans une population d'enfants et huit dans des populations d'adultes. Des trois études présentant des données probantes de la plus haute qualité (niveau I), une a démontré des avantages pharmacodynamiques de la perfusion continue de vancomycine. Des six études présentant des données probantes de qualité modérée (niveau II), trois ont aussi démontré des avantages liés à la perfusion continue sur les plans pharmacocinétique et pharmacodynamique, mais les conclusions en termes de résultats cliniques étaient mitigées.

Conclusions : Les données actuelles sur l'évaluation des avantages pharmacocinétiques et pharmacodynamiques et l'efficacité clinique de la perfusion i.v. continue par rapport à la perfusion i.v. intermittente de vancomycine sont contradictoires et ne justifient pas le recours systématique à la perfusion continue pour le traitement des infections à bactéries Gram-positif multirésistantes aux antibiotiques.

Mots clés : vancomycine, perfusion continue, perfusion intermittente

[Traduction par l'éditeur] 


\section{INTRODUCTION}

Tancomycin is the mainstay of treatment for multidrugresistant gram-positive organisms, including methicillinresistant Staphylococcus aureus (MRSA) and methicillinresistant coagulase-negative Staphylococcus (MRCNS). Vancomycin exhibits concentration-independent activity, whereby high peak concentrations are not necessary to achieve bacterial killing. Rather, it is thought that serum concentration of the drug should be maintained at 4 to 5 times the minimal inhibitory concentration (MIC) for bactericidal activity. ${ }^{1}$ Historically, on the basis of pharmacokinetic studies and anecdotal reports, vancomycin dosing was targeted to achieve peak serum concentrations of $30-40 \mathrm{mg} / \mathrm{L}$ and trough concentrations of 5-10 $\mathrm{mg} / \mathrm{L} .{ }^{2}$ More recent clinical guidelines have recommended target trough concentrations of $15-20 \mathrm{mg} / \mathrm{L}$ for the treatment of complicated MRSA infections, including bacteremia, meningitis, osteomyelitis, hospital-acquired pneumonia, and endocarditis. ${ }^{3}$ Trough concentrations less than $10 \mathrm{mg} / \mathrm{L}$ are associated with the development of vancomycinintermediate $S$. aureus strains. ${ }^{3}$ However, even when a trough level of $15 \mathrm{mg} / \mathrm{L}$ is achieved, clinical outcomes do not appear to improve in patients infected with $S$. aureus strains exhibiting an MIC of $2 \mathrm{mg} / \mathrm{L}$ or greater. ${ }^{4}$ Furthermore, values for the ratio of area under the concentration-time curve to minimum inhibitory concentration (AUC/MIC) of 400 or greater have been most predictive of clinical efficacy. ${ }^{3}$ However, no studies have demonstrated correlation between trough concentration of $15-20 \mathrm{mg} / \mathrm{L}$ and AUC/MIC greater than 400 .

To achieve the higher recommended serum concentration targets, vancomycin dosages of $15-20 \mathrm{mg} / \mathrm{kg}$ every $4-12 \mathrm{~h}$, depending on the patient's age and renal function, may be required. Some have proposed that continuous IV infusion would be a better dosing strategy to achieve higher serum concentrations of the drug.

The theoretical advantages of continuous administration of vancomycin include the ability to consistently maintain drug concentrations above the MIC, without large fluctuations. This may minimize dips in serum concentrations at the end of the dosing interval and avoid potential toxic effects associated with high peak concentrations. However, the correlation between high vancomycin concentrations in serum and nephrotoxicity and ototoxicity is poor. ${ }^{3,5}$

Pharmacokinetic studies have demonstrated shorter time to and longer duration of target serum concentrations with continuous infusion than with intermittent administration for equivalent total daily doses. ${ }^{6-8}$ However, with continuous infusion, the concentration of vancomycin penetrating into lung tissue was not superior to that achieved by intermittent dosing.' Notably, these theoretical benefits have been derived solely from pharmacokinetic studies (but there were methodologic inconsistencies across these studies, which limited their comparability); whether they will improve clinical outcomes requires evaluation by randomized controlled trials. As such, the ability of continuous infusion to generate higher serum concentrations and better antimicrobial activity against deeptissue infections remains a subject of debate.

In some studies, use of a continuous-infusion strategy necessitated fewer serum samples for appropriate dosage adjustments. ${ }^{7.8}$ Other studies have suggested a lower total dose requirement, decreased pharmacy compounding time, and decreased nursing time. ${ }^{10}$ However, these potential advantages have not been confirmed in clinical trials.

The purpose of this review was to systematically assess the literature comparing continuous infusion and conventional intermittent IV dosing for administration of vancomycin, in terms of efficacy and safety.

\section{METHODS}

\section{Data Sources}

A literature search was conducted in the PubMed/MEDLINE (1950 to January 2010) and Embase (1980 to January 2010) databases and the Cochrane Central Register of Controlled Trials. The Internet was searched by means of the Google search engine, and the reference lists of relevant articles were searched manually. The search terms included "vancomycin", "continuous infusion", "constant rate", "intermittent infusion", "discontinuous infusion", and "administration".

\section{Selection of Studies}

Pharmacokinetic, pharmacodynamic, and clinical studies were included if they compared continuous and intermittent IV administration of vancomycin. There were no restrictions on age of patients or quality of the trials. Studies with the following characteristics were excluded: nonhuman data, not published in English or French, published as abstract only, absence of a comparator arm with intermittent dosing, and assessment of intraperitoneal administration.

\section{Extraction and Evaluation of Data}

Studies were categorized according to the levels of evidence outlined in the US Preventive Services Task Force rating scale. ${ }^{11}$ The levels of evidence ranged from I, for evidence obtained from at least one properly randomized controlled trial, to III, for opinions of respected authorities, descriptive studies and case reports, or reports of expert committees. The data extracted from each study included the following information: study design, number of participants, characteristics of the study population, exclusion criteria, drug dosing regimens, outcome measures, and study conclusions.

\section{RESULTS}

A total of 9 studies met the inclusion criteria and were included in this systematic review (Table 1)..$^{7,812-18}$ 


\section{Level I Evidence}

Three studies of very different methodologic designs represented the highest level of evidence, properly randomized controlled trials. ${ }^{8,12,13}$ Although classified as level I evidence because the study participants were randomly assigned to treatment groups, ${ }^{11} 2$ of these studies ${ }^{12,13}$ evaluated pharmacokinetic and pharmacodynamic outcomes rather than clinical outcomes. The results of these studies are informative on issues related to antibiotic activity and pharmacodynamics, but their nature is entirely different from that of results obtained in clinical efficacy trials.

James and others ${ }^{12}$ conducted a prospective, randomized, crossover study involving 10 patients with suspected or documented gram-positive infections (2 patients with bacteremia, 2 with line infection, 2 with osteomyelitis, and 1 each with urinary tract infection, pneumonia, brain abscess, and cellulitis). To determine serum bactericidal titres, the authors used $2 S$. aureus isolates: one sensitive and the other resistant to methicillin. The serum bactericidal titre, reflecting the organisms' in vitro susceptibility, was defined as the greatest dilution that resulted in a $99.9 \%$ reduction in microbial growth compared with the starting inoculum. The MICs were $0.78 \mathrm{mg} / \mathrm{L}$ for methicillin-sensitive $S$. aureus and 1.56 $\mathrm{mg} / \mathrm{L}$ for the MRSA isolate. Both continuous and intermittent dosing resulted in time above MIC ( $T>_{\text {MIC }}$ ) of $100 \%$ for both $S$. aureus isolates. No adverse effects or significant changes in serum creatinine were observed in either group.

Klepser and others ${ }^{13}$ conducted a pharmacokinetic and pharmacodynamic crossover study in 12 healthy volunteers who were randomly assigned to receive 1 of 3 treatment regimens (as summarized in Table 1). Samples were collected before dosing and at $1,2,4,6,8,10$, and $12 \mathrm{~h}$ after administration of the antibiotic. Serum inhibitory and bactericidal titres for each treatment regimen were calculated using 2 MRSA isolates (with MICs of 1.0 and $4.0 \mathrm{mg} / \mathrm{L}$, respectively). All 3 regimens resulted in 100\% coverage for the MRSA isolate with MIC of $1.0 \mathrm{mg} / \mathrm{L}$. For the isolate with MIC of $4.0 \mathrm{mg} / \mathrm{L}$, continuous infusion (either $1 \mathrm{~g} /$ day or $2 \mathrm{~g} /$ day) resulted in measurable bactericidal activity for $100 \%$ of the infusion period (compared with mean 98.5\% \pm standard deviation $4.9 \%$ with intermittent dosing of $1 \mathrm{~g} \mathrm{q} 12 \mathrm{~h}$; $p$ value not reported). Overall, the study drug, which was administered to all patients through a peripheral vein, was poorly tolerated. All patients reported thrombophlebitis, the most severe cases occurring in the group that received vancomycin $2 \mathrm{~g} /$ day by continuous infusion.

The third study with level I evidence ${ }^{8}$ was the largest in this category, involving 119 critically ill patients with staphylococcal infections (81\% with MRSA, 19\% with coagulasenegative Staphylococcus). The patients had a variety of illnesses at baseline, most commonly pneumonia (48\%) and bacteremia (44\%). For patients randomly assigned to undergo intermittent dosing, target trough concentrations between 10 and $15 \mathrm{mg} / \mathrm{L}$ were prespecified; for those undergoing continuous infusion, plateau concentrations were targeted to $20-25 \mathrm{mg} / \mathrm{L}$. Overall mortality was $18 \%$ among patients in the continuous infusion group and $12 \%$ in the intermittent infusion group. However, infection-related deaths, as determined by a committee blinded to infusion mode, were $10 \%$ and $12 \%$, respectively. Upon completion of the study, failure of therapy (defined as death from infection or lack of change in or worsening of clinical, laboratory, and radiological status relative to day 0) had occurred for $54 \%$ of patients receiving continuous infusion and $46 \%$ of those undergoing intermittent infusion. No $p$ values were reported for these outcomes because the study had inadequate power (only 23\%) for such statistical comparisons. Continuous infusion of vancomycin resulted in a shorter time to reach target serum concentrations (mean \pm standard deviation $36 \pm 31 \mathrm{~h}$ versus $51 \pm 39 \mathrm{~h}, p=0.03)$, and fewer serum samples were required for monitoring $(7.7 \pm 2.2$ versus $11.8 \pm 3.9, p<0.0001)$. Serum vancomycin concentrations were measured daily until 2 consecutive readings within the targeted range were achieved. Nephrotoxicity, defined as a 50\% increase in serum creatinine, was observed in $16 \%$ of the patients undergoing continuous infusion and 19\% of those undergoing intermittent infusion $(p=0.64)$. However, the need for hemodialysis was twice as high in the group undergoing continuous infusion (10\% versus 5\%). Accounting only for direct drug costs and the cost of determining serum concentration of vancomycin, continuous infusion was associated with an average cost saving of US $\$ 133$ per 10 -day course $(p<0.0001)$.

Summary: Among the studies with the highest level of evidence, there was inconsistency regarding whether continuous infusion of vancomycin offered pharmacodynamic advantages over traditional intermittent dosing. In 2 of the studies, both regimens achieved serum concentrations above the MIC $98.5 \%$ or $100 \%$ of the time..$^{12,13}$ In the third study, continuous infusion was associated with a shorter time to achieve target serum concentrations and fewer sample collections for dosing adjustments. ${ }^{8}$ Two of these studies involved small numbers of participants, and they evaluated surrogate outcomes rather than clinical end points. ${ }^{12,13}$ In addition, continuous infusion was not associated with improved bactericidal activity against MRSA strains with MICs of 1.0 and $4.0 \mathrm{mg} / \mathrm{L} .{ }^{13}$ In the study involving critically ill patients, ${ }^{8}$ rates of mortality and treatment failure were higher with continuous infusion, although the study was not sufficiently powered to formally evaluate these outcomes. The rates of adverse events associated with each dosing regimen were conflicting between studies. Continuous infusion was associated with lower costs in the study that examined this aspect of therapy. ${ }^{8}$

\section{Level II-1 Evidence}

The literature search identified one well-designed controlled trial without randomization. This prospective, multicentre study compared continuous and intermittent 


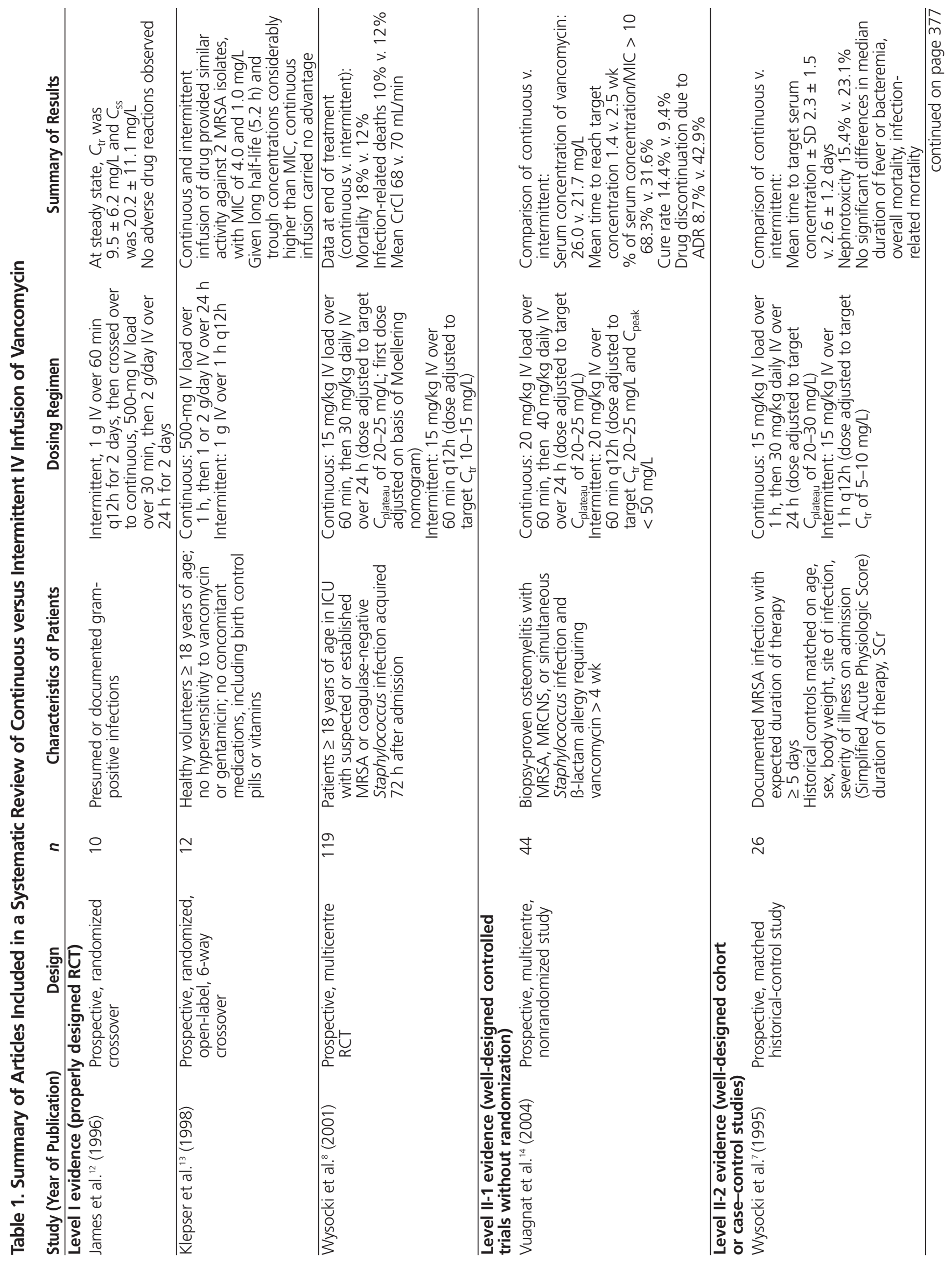




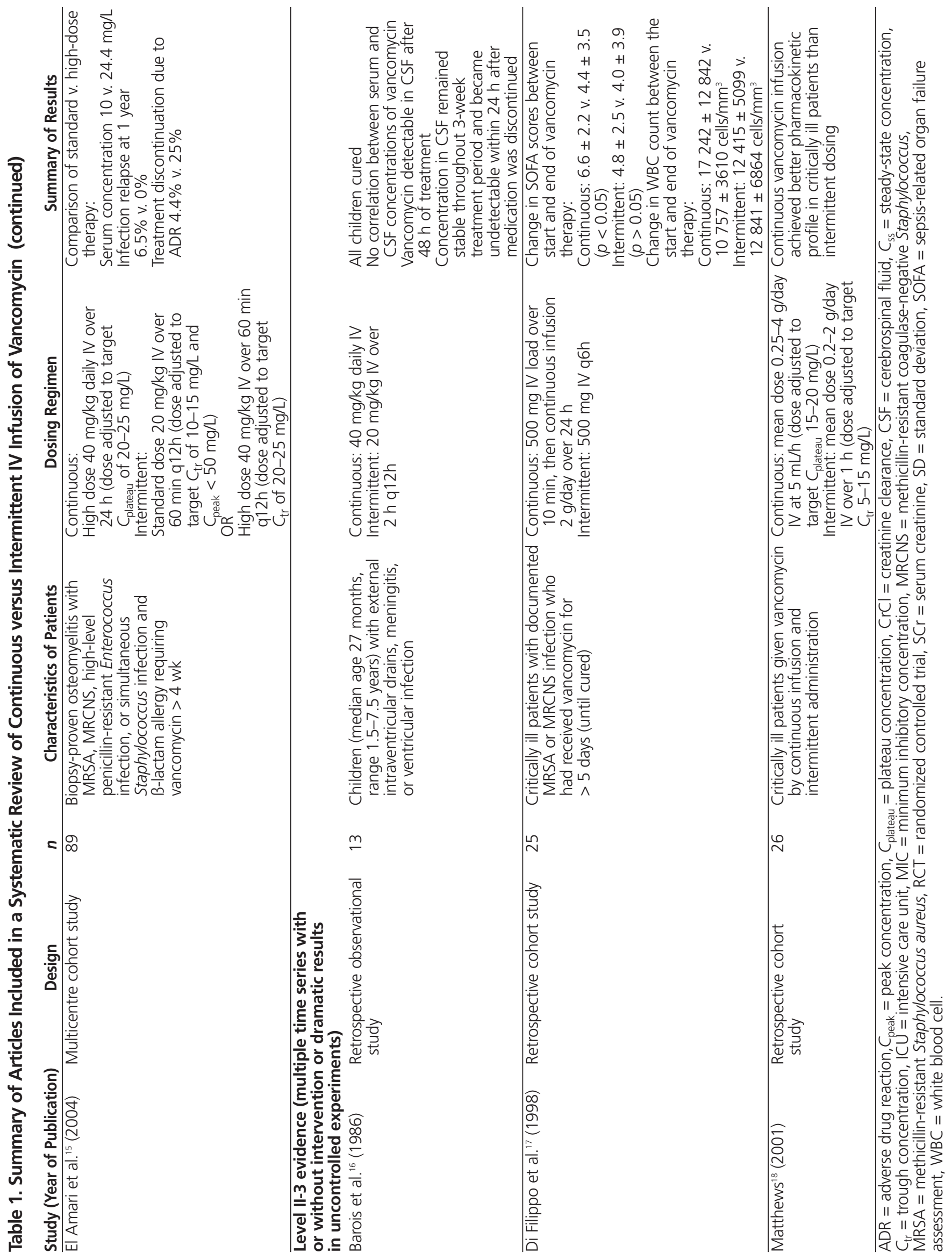


vancomycin therapy in terms of efficacy and safety in 44 patients with chronic osteomyelitis. ${ }^{14}$ MRSA and MRCNS were isolated in $71 \%$ and $28 \%$ of patients, respectively. MICs ranged from 1.0 to $4.0 \mathrm{mg} / \mathrm{L}$. Vancomycin was initiated during the hospital stay and was continued as parenteral antimicrobial therapy in an outpatient setting (mean total treatment duration 12.5 weeks). Fourteen of the patients (5 receiving continuous and 9 receiving intermittent vancomycin therapy) received concomitant rifampin, and 6 of the patients ( 4 receiving continuous and 2 receiving intermittent vancomycin therapy) received concomitant ciprofloxacin.

The difference in cure rate (defined as absence of recurrent symptoms for 12 months after completion of therapy) was not statistically significant ( $94 \%$ with continuous therapy versus $78 \%$ with intermittent therapy; $p=0.25$ ). However, the study was probably underpowered to detect a significant difference in this outcome. Also, given the unblinded nature of the study, patients with more severe infections may have been more likely to undergo continuous administration of vancomycin. The rates of discontinuation secondary to treatment failure (defined as persistent symptoms at infection site or recurrence of infection despite antibiotic therapy) were also similar: $8.7 \%$ with continuous therapy versus $9.5 \%$ with intermittent therapy ( $p$ value not specified). Likewise, the time to reach target vancomycin concentration and the number of dosing adjustments did not differ significantly between the groups. However, the discontinuation rate due to adverse drug reactions was markedly lower in patients undergoing continuous dosing $(8.7 \%$ versus $42.9 \%, p=0.03)$. None of the patients receiving vancomycin by continuous infusion experienced a $50 \%$ increase in serum creatinine, but this did occur for $19 \%$ of the patients receiving vancomycin by intermittent dosing. In addition, $95 \%$ of the patients undergoing continuous infusion but only $57 \%$ of those in the intermittent dosing group were free of adverse reactions by week $6(p=0.005)$. After adjustment for potential confounders, only 2 independent factors were associated with adverse drug reactions leading to termination of therapy: intermittent dosing (relative risk [RR] $=5.9, p=0.003)$ and osteomyelitis of the foot $(\mathrm{RR}=5.2, p=0.01)$.

Summary: In a prospective, nonrandomized trial involving patients with chronic staphylococcal osteomyelitis, there was no statistically significant improvement in cure rates with continuous infusion of vancomycin relative to intermittent dosing. However, the study was probably insufficiently powered to evaluate this outcome. Compared with patients undergoing continuous infusion, those treated with intermittent doses experienced significantly higher rates of adverse effects necessitating early discontinuation of therapy.

\section{Level II-2 Evidence}

Two well-designed cohort or case-control studies were identified, and these were categorized as providing level II-2 evidence. ${ }^{7,15}$
The first study ${ }^{7}$ compared 13 patients prospectively treated with vancomycin by continuous infusion with 13 matched historical controls treated with intermittent infusion for severe MRSA infections. Matching was 73\% successful for the 7 prespecified parameters (listed in Table 1). Patients were matched completely with regard to sex, body weight, site of infection, and duration of therapy. However, those receiving continuous infusion were younger $(61 \pm 17$ years versus $67 \pm$ 13 years) and had a greater severity of illness, as indicated by the higher Simplified Acute Physiologic Score $(17 \pm 5$ versus $13 \pm$ 3). Baseline serum creatinine measurements were not reported. There were no statistically significant differences between the groups in overall morality rate, infection-related mortality rate, median duration of fever, median duration of bacteremia, or change in serum creatinine. Continuous infusion resulted in shorter time to target serum concentrations of vancomycin ( $2.3 \pm 1.5$ days versus $2.6 \pm 1.2$ days $)$ and fewer measurements of serum concentration ( $9 \pm 5$ versus $10 \pm 7$ ), but the statistical significance of these differences was not reported. Nephrotoxicity (defined as a rise in serum creatinine by at least 44.2 $\mu \mathrm{mol} / \mathrm{L}$ if baseline value was less than or equal to $265.2 \mu \mathrm{mol} / \mathrm{L}$ or a rise by at least $88.4 \mu \mathrm{mol} / \mathrm{L}$ if baseline value was above $265.2 \mu \mathrm{mol} / \mathrm{L}$ ) was observed in 2 patients who underwent continuous infusion and 3 patients who underwent intermittent infusion.

The second study ${ }^{15}$ enrolled 89 patients with biopsyproven osteomyelitis who required prolonged vancomycin therapy. Forty-five of the patients received standard-dose therapy (20 mg/kg daily) given intermittently, and 44 received high-dose therapy (40 mg/kg daily) given by continuous $(n=23)$ or intermittent $(n=21)$ infusion. All patients underwent surgical debridement or removal of infected prosthetic material before initiation of the antibiotic. Rates of treatment failure and recurrence of infection were compared between standard- and high-dose vancomycin, but the data for intermittent and continuous dosing within the high-dose group were not reported separately. Kaplan-Meier analysis of pooled outcomes (treatment effect without beneficial outcomes, adverse drug reactions, and treatment failure) showed superiority of high-dose therapy by continuous infusion over standarddose therapy and over high-dose therapy by intermittent administration (log rank $p=0.02)$.

Summary: In a matched historical-control study, ${ }^{7}$ continuous infusion of vancomycin was not associated with improvements in overall mortality, infection-related mortality, or duration of bacteremia. Although continuous infusion allowed target serum concentrations to be reached earlier and allowed the collection of fewer serum samples, the differences were probably of negligible importance. In a second study, ${ }^{15}$ high-dose vancomycin therapy by continuous infusion led to fewer adverse drug reactions and treatment failures relative to standard-dose and high-dose therapy by intermittent infusion, although the results were not explicitly reported. 


\section{Level II-3 Evidence}

Three studies with level II-3 evidence (i.e., evidence from multiple time series with or without intervention or dramatic results in uncontrolled experiments) were identified. All three studies were retrospective. One involved a pediatric population ${ }^{16}$ and 2 involved adult patients. ${ }^{17,18}$

The concentration of vancomycin in cerebrospinal fluid (CSF) and plasma was evaluated in 13 children receiving the drug by continuous infusion for staphylococcal meningitis $(n=3)$ and infections of a ventricular shunt $(n=10) ; 2$ of the children had initially received vancomycin by intermittent dosing but were later switched to continuous dosing. ${ }^{16}$ Twelve of the patients had coagulase-negative Staphylococcus infections, and one was infected with $S$. aureus. In the 2 children (aged 17 months and 14 years) treated initially by intermittent administration $(20 \mathrm{mg} / \mathrm{kg}$ IV $\mathrm{q} 12 \mathrm{~h}$ infused over $2 \mathrm{~h})$, the serum concentrations of vancomycin were $9 \mathrm{mg} / \mathrm{L}$ (trough) and $21 \mathrm{mg} / \mathrm{L}$ (peak), whereas the drug was undetectable in the CSF. The duration of intermittent therapy was not described, and these 2 patients were eventually switched to continuous infusion. After $48 \mathrm{~h}$ of vancomycin therapy by continuous infusion, the CSF concentration of the drug in both children was above $1 \mathrm{mg} / \mathrm{L}$.

Six of the children received vancomycin by continuous infusion from the beginning of therapy and underwent serial determinations of vancomycin in the serum and CSF during the initial $48 \mathrm{~h}$ of treatment. For these children, vancomycin concentration was detectable in the CSF at $48 \mathrm{~h}$. The drug concentration remained stable between day 3 and cessation of therapy (range 1 to $3.2 \mathrm{mg} / \mathrm{L}$ ), with a median duration of therapy of 3 weeks (range 1 to 4 weeks). Vancomycin became undetectable in the CSF within $24 \mathrm{~h}$ of discontinuation. The concentration of vancomycin in plasma ranged from 6 to $50 \mathrm{mg} / \mathrm{L}$.

In all 13 patients, no correlation was found between plasma $(6-50 \mu \mathrm{g} / \mathrm{mL})$ and CSF $(1-3.2 \mu \mathrm{g} / \mathrm{mL})$ concentrations of the drug (based on 23 plasma and CSF samples drawn concurrently). No complications were associated with continuous infusion of vancomycin, although renal function and adverse events were not reported. All 13 patients survived and were considered cured. One child experienced a recurrence 1 month after discontinuation of therapy.

A retrospective cohort study compared the efficacy of the 2 dosing regimens in 25 critically ill adults with MRSA or MRCNS infection, specifically evaluating the Sepsis-Related Organ Failure Assessment (SOFA) score and white blood cell (WBC) count before and after treatment. ${ }^{17}$ Mean patient ages were $70 \pm 14$ years (continuous therapy) and $63 \pm 24$ years (intermittent therapy). Two patients in each group had additional mixed infections (Klebsiella pneumoniae and Pseudomonas in both) and received concomitant therapy with monobactams or aminoglycosides. Mean baseline SOFA scores were $6.6 \pm 2.2$ (continuous therapy) and $4.8 \pm 2.5$ (intermittent therapy). The mean SOFA score at the end of therapy was significantly lower than baseline in the group that underwent continuous infusion $(4.4 \pm 3.5 ; p<0.05)$ but was not significantly different from baseline in the group that underwent intermittent dosing $(4.0 \pm 3.9 ; p>0.05)$. The changes in WBC count after institution of vancomycin therapy were also significant in the continuous infusion group (17 242 \pm 12842 cells $/ \mathrm{mm}^{3}$ before therapy versus $10757 \pm 3610$ cells $/ \mathrm{mm}^{3}$ after therapy) but not in the intermittent dosing group (12 $415 \pm$ 5099 cells $/ \mathrm{mm}^{3}$ before therapy versus $12841 \pm 6864$ cells $/ \mathrm{mm}^{3}$ after therapy). Mean serum concentrations of vancomycin were compared between continuous and intermittent dosing, but the statistical significance was not reported (at $2 \mathrm{~h}, 13.9 \pm 5.7$ versus $15.0 \pm 3.5 \mathrm{mg} / \mathrm{L}$; at $48 \mathrm{~h}, 17.8 \pm 7.6 \mathrm{mg} / \mathrm{L}$ versus 22.5 $\pm 5.6 \mathrm{mg} / \mathrm{L}$; and at $96 \mathrm{~h}, 4.3 \pm 3.9$ versus $30.7 \pm 6.4 \mathrm{mg} / \mathrm{L}$ ). No adverse effects were observed in either group. Interestingly, patients assigned to undergo continuous dosing were given an initial dose of $500 \mathrm{mg}$ IV over $10 \mathrm{~min}$. Such rapid administration of vancomycin may increase the risk of infusion-related adverse effects. ${ }^{19}$

The second study involving adults evaluated 26 patients in whom the MIC of vancomycin for all sensitive organisms isolated was less than $4 \mathrm{mg} / \mathrm{L} .{ }^{18}$ The total daily doses used were highly variable among patients and between the 2 dosing regimens $(2.5-4 \mathrm{~g} /$ day for continuous infusion and $0.2-2$ $\mathrm{g} /$ day for intermittent dosing). Twenty-two of the 23 patients who received vancomycin by both modes of administration had higher serum concentrations of the drug with continuous infusion; the one outlier actually received a lower daily dose during the continuous infusion course than the intermittent dosing course. All patients achieved serum concentrations above the MIC with continuous infusion, whereas 4 patients had concentrations below the MIC with intermittent dosing. Three patients experienced adverse drug reactions while receiving vancomycin by continuous infusion (a rash in 2 patients, interstitial nephritis in 1 patient); no adverse reactions were observed during intermittent infusion.

Summary: A pediatric study reported continuous and intermittent administration of vancomycin to children with staphylococcal meningitis and ventricular shunt infections. ${ }^{16}$ Only administration by continuous infusion resulted in detectable concentrations of the drug in the CSF, and there was no correlation between vancomycin concentrations in the serum and the CSF. In a study of critically ill adults, ${ }^{17}$ there was significant improvement in SOFA scores and reduction in WBC counts with continuous infusion of vancomycin, but no significant changes were observed in the intermittent dosing group. However, patients in the continuous dosing group had higher SOFA scores and WBC counts at baseline, which signifies a potentially sicker population than the control group. No adverse effects were reported in either group. In a third study, ${ }^{18}$ adult patients treated with vancomycin by continuous infusion achieved higher serum concentrations and consistently 
maintained concentrations above the MIC. However, the dosing and sampling strategies were highly variable.

\section{DISCUSSION}

Limited evidence is currently available to evaluate the clinical efficacy and safety of vancomycin by continuous infusion relative to conventional intermittent infusion. Published trials were highly heterogeneous in terms of study design, dosing regimens, and serum monitoring strategies, and evaluated a vast array of outcomes that rendered comparison across trials difficult. Although both adult and pediatric populations were of interest, only one pediatric study met the inclusion criteria for this review.

A common limitation to the existing literature is the relatively small sample populations used for individual studies. Sample size was generally less than 30 , and the largest trial involved only 119 patients. ${ }^{8}$ That randomized controlled trial is the largest comparison of continuous and intermittent administration of vancomycin published to date and has been frequently cited by other authors. However, because it did not meet the prespecified sample size of 320 (and thus had only $23 \%$ power to assess its primary outcome), ${ }^{8}$ its results should be interpreted with caution. Unfortunately, that study also used different prespecified target concentrations of vancomycin for the intermittent and continuous infusion groups. Therefore, the results reflected not only the 2 modes of vancomycin administration but also a discrepancy in target serum concentrations. The other 8 studies that were identified and analyzed here did not report a priori sample size calculations. It was also unclear whether they were designed to be superiority, noninferiority, or equivalence studies.

Some studies assessed only pharmacokinetic and pharmacodynamic outcomes. ${ }^{12,13,18}$ Although continuous infusion was hypothesized to shorten the time to reach target serum concentrations (defined as time of administration of the first dose to time when target concentration is reached) and lengthen the $T_{>\mathrm{MIC}}$ at equivalent doses, these assumptions were not supported by 2 of the 3 studies. ${ }^{12,13}$ Moreover, a shorter time to target concentration is not a compelling benefit because it can be achieved with a loading dose, regardless of the dosing regimen.

Other studies included in this review focused on whether continuous vancomycin dosing would improve clinical efficacy and safety rather than on surrogate pharmacokinetic outcomes. ${ }^{7,8,14-17}$ Two prospective controlled trials were identified, one randomized controlled trial involving critically ill patients with a variety of MRSA and MRCNS infections (level I evidence) $)^{8}$ and one nonrandomized controlled trial in patients with chronic MRSA and MRCNS osteomyelitis (level II-1 evidence). ${ }^{14}$ In neither study were there significant differences in rates of treatment failure, overall mortality, or infection-related mortality between the 2 treatment regimens.
As noted earlier, one of these studies was significantly underpowered to assess treatment failure, ${ }^{8}$ and the other did not report the required sample size a priori ${ }^{14}$ so it remains unclear whether it had adequate power to assess cure rate.

A study of chronic osteomyelitis was designed primarily to evaluate standard-dose versus high-dose vancomycin, with the high-dose group further divided into continuous and intermittent dosing. ${ }^{15}$ Unfortunately, the authors did not report comparative data for continuous and intermittent administration of high-dose therapy, and the data pertaining to continuous dosing were limited, both of which restrict the contribution of this study to the analysis presented here. The other retrospective study revealed a link between continuous infusion and higher frequency of serum concentrations above the MIC. ${ }^{18}$ However, many potential confounding factors might have affected serum concentrations of vancomycin, including dosage, serum sampling times, pathogens isolated and their level of resistance, and experience of the clinicians making the dosage adjustments. Only the lowest recorded serum concentrations of vancomycin were reported, so these data did not capture whether target concentrations were sustained throughout the course of treatment or whether supratherapeutic concentrations were observed.

Only one study evaluated the cost-effectiveness of the 2 dosing regimens, estimating a cost saving of US $\$ 133$ per 10-day course with the continuous infusion regimen. ${ }^{8}$ This saving may be negligible in light of the substantial costs associated with admission to hospital and related care. It would be useful to assess the satisfaction of nurses and patients, potential administration challenges (e.g., line access, IV compatibility), and receptiveness in the outpatient setting for these 2 modes of administration. It might be argued that continuous infusion is more labour-intensive, but this end point is highly subjective. It is also important to note that cost estimates should be interpreted with caution as they are subject to variation according to dosage adjustments and serum monitoring protocols of the institution.

Overall, the studies included in this review had inconsistent findings. They were highly heterogeneous in terms of methodologic design, patient population, dosing and sampling strategies, and outcomes evaluated. Common limitations to these studies were small sample size, poor reporting of adverse drug reactions, and insufficient consideration of potential confounding factors that might bias outcomes. For example, timely and accurate diagnosis and initiation of antibiotics have been shown to improve mortality in the intensive care unit. ${ }^{20}$ Potential confounders, such as severity of illness, comorbid conditions, multipathogen infections, and other interventions (e.g., surgical debridement), were seldom addressed in the current literature but are likely to affect outcome measures to a larger extent than would the mode of vancomycin administration. In light of these limitations, there appears to be a lack of sound evidence to support the use of continuous infusion of 
vancomycin for treating multidrug-resistant gram-positive infections. Furthermore, continuous infusion does not appear to reduce costs relative to traditional intermittent dosing.

This systematic review had some limitations. Only articles published in English and French were included. As a result, 3 potentially relevant articles ( 2 in Spanish and 1 in Japanese) were excluded. Only studies that directly compared continuous with intermittent vancomycin infusion were eligible for review. Studies evaluating pharmacokinetic characteristics and clinical efficacy of continuous infusion alone were not included.

\section{CONCLUSIONS}

Current evidence does not support routine administration of vancomycin by continuous infusion for various multidrugresistant gram-positive infections. Although this review does not negate the potential role of continuous vancomycin infusion in unique therapeutic circumstances, such specific cases warrant individualized clinical judgement based on patient-specific parameters and evaluation of the potential benefits and risks of each administration modality.

\section{References}

1. Roberts JA, Lipman J, Blot S, Rello J. Better outcomes through continuous infusion of time-dependent antibiotics to critically ill patients? Curr Opin Crit Care 2008;14(4):390-396.

2. Geraci JE. Vancomycin. Mayo Clin Proc 1977;52(10):631-634.

3. Rybak MJ, Lomaestro B, Rotschafer JC, Moellering R Jr, Craig W, Billeter $\mathrm{M}$, et al. Therapeutic monitoring of vancomycin in adult patients: a consensus review of the American Society of Health-System Pharmacists, the Infectious Diseases Society of America, and the Society of Infectious Diseases Pharmacists. Am J Health Syst Pharm 2009;66(1):82-98. Erratum in: Am J Health Syst Pharm 2009;66(10):887.

4. Hidayat LK, Hsu DI, Quist R, Shriner KA, Wong-Beringer A. High-dose vancomycin therapy for methicillin-resistant Staphylococcus aureus infections: efficacy and toxicity. Arch Intern Med 2006;166(19):2138-2144.

5. Leader WG, Chandler MH, Castiglia M. Pharmacokinetic optimisation of vancomycin therapy. Clin Pharmacokinet 1995;28(4):327-342.

6. Watanabe T, Ohashi K, Matsui K, Kubota T. Comparative studies of the bactericidal, morphological and post-antibiotic effects of arbekacin and vancomycin against methicillin-resistant Staphylococcus aureus. J Antimicrob Chemother 1997;39(4):471-476.

7. Wysocki M, Thomas F, Wolff MA, Pean Y, Ravaud Y, Herman B. Comparison of continuous with discontinuous intravenous infusion of vancomycin in severe MRSA infections. $J$ Antimicrob Chemother 1995;35(2):352-354.

8. Wysocki M, Delatour F, Faurisson F, Rauss A, Pean Y, Misset B, et al. Continuous versus intermittent infusion of vancomycin in severe staphylococcal infections: prospective multicenter randomized study. Antimicrob Agents Chemother 2001;45(9):2460-2467.

9. Byl B, Jacobs F, Wallemacq P, Rossi C, de Francquen P, Cappello M, et al. Vancomycin penetration of uninfected pleural fluid exudate after continuous or intermittent infusion. Antimicrob Agents Chemother 2003;47(6):20152017.

10. Ronchera-Oms CL, Gregorio S, Sanllehí N. Should continuous infusion of B-lactam antibiotics be the first-line approach? J Clin Pharm Ther 1997;22(3):159-161.
11. U.S. Preventive Services Task Force. Guide to clinical preventive services: report of the U.S. Preventive Services Task Force. 2nd ed. Baltimore (MD): Williams \& Wilkins; 1996.

12. James JK, Palmer SM, Levine DP, Rybak MJ. Comparison of conventional dosing versus continuous-infusion vancomycin therapy for patients with suspected or documented gram-positive infections. Antimicrob Agents Chemother 1996;40(3):696-700.

13. Klepser M, Patel K, Nicolau DP, Quintiliani R, Nightingale $\mathrm{CH}$. Comparison of bactericidal activities of intermittent and continuous infusion dosing of vancomycin against methicillin-resistant Staphylococcus aureus and Enterococcus faecalis. Pharmacotherapy 1998;18(5): 1069-1074.

14. Vuagnat A, Stern R, Lotthe A, Schuhmacher H, Duong M, Hoffmeyer P, et al. High dose vancomycin for osteomyelitis: continuous vs. intermittent infusion. J Clin Pharm Ther 2004;29(4):351-357.

15. El Amari EB, Vuagnat A, Stern R, Assal M, Denormandie P, Hoffmeyer $\mathrm{P}$, et al. High versus standard dose vancomycin for osteomyelitis. Scand J Infect Dis 2004;36(10):712-717.

16. Barois A, Estournet B, Moranne JB, Piliot J, Chabenat C, Bataille J. [Ventricular staphylococcal infections. Treatment with vancomycin by continuous venous infusion]. Presse Med 1986;15(36):1805-1808. Article in French.

17. Di Filippo A, De Gaudio AR, Novelli A, Paternostro E, Pelagatti C, Livi $P$, et al. Continuous infusion of vancomycin in methicillin-resistant staphylococcus infection. Chemotherapy 1998;44(1):63-68.

18. Matthews Z. Vancomycin continuous infusion: a cohort of 23 intensive care unit patients. Aust J Hosp Pharm 2001;31(2):108-110.

19. Sivagnanam S, Deleu D. Red man syndrome. Crit Care 2003; 7(2):119-120.

20. Rivers E, Nguyen B, Havstad S, Ressler J, Muzzin A, Knoblich B, et al.; Early Goal-Directed Therapy Collaborative Group. Early goal-directed therapy in the treatment of severe sepsis and septic shock. N Engl J Med 2001;345(19):1368-1377.

Sally S K Man, BSc(Pharm), PharmD, is Provincial Pharmacy Education Coordinator, BC Cancer Agency, Vancouver British Columbia. At the time of writing, she was a Doctor of Pharmacy student in the Faculty of Pharmaceutical Sciences, The University of British Columbia, Vancouver, British Columbia.

Roxane R Carr, PharmD, BCPS, is Clinical Leader, Pharmacy Critical Care Services, Department of Pharmacy, Children's and Women's Health Centre of British Columbia and Assistant Professor, part-time, Faculty of Pharmaceutical Sciences, The University of British Columbia, Vancouver, British Columbia.

Mary H H Ensom, PharmD, FASHP, FCCP, FCSHP, FCAHS, is Professor and Director, Doctor of Pharmacy Program, Faculty of Pharmaceutical Sciences, and Distinguished University Scholar, The University of British Columbia, and Clinical Pharmacy Specialist, Department of Pharmacy, Children's and Women's Health Centre of British Columbia, Vancouver, British Columbia. She is also the Editor of the CJHP.

\section{Address correspondence to:}

Dr Mary $\mathrm{H} \mathrm{H}$ Ensom

Department of Pharmacy (OB7)

Children's and Women's Health Centre of British Columbia 4500 Oak Street

Vancouver BC V6H 3N1

e-mail: ensom@interchange.ubc.ca 